\title{
RESEARCH ON MANAGEMENT AND APPLICATION OF TUNNEL ENGINEERING BASED ON BIM TECHNOLOGY
}

\author{
ZhanPing SONG $1,2,3^{*}$, GuiLin SHI $1,2,3$, JunBao WANG ${ }^{1,2,3^{\#}}$, \\ HaiMin WEI ${ }^{1,2,3}$, Tao WANG ${ }^{2,4}$, GuanNan ZHOU ${ }^{2,4}$ \\ ${ }^{1}$ School of Civil Engineering, Xian University of Architecture and Technology, Xian 710055, China \\ ${ }^{2}$ Shaanxi Key Laboratory of Geotechnical and Underground Space Engineering, Xian 710055, China \\ ${ }^{3}$ Institute of Tunnel and Underground Structure of Xian University of Architecture and Technology, \\ Xian 710055, China \\ ${ }^{4}$ China Railway Construction Bridge Engineering Bureau Group Co., Ltd. Tianjin 300300, China
}

Received 30 April 2019; accepted 05 June 2019

\begin{abstract}
The emergence of BIM technology has provided powerful technical means for realizing informatization and digitization in the field of engineering construction, which remarkably promotes the transformation and advance of production and management modes in engineering construction. Presently, the application and development of BIM technology in the field of engineering construction has become increasingly mature, yet in the field of tunnel engineering, the application of BIM technology is still in its infancy. Under such a circumstance, this paper first puts forward the basic hardware and software configuration requirements for BIM technology in tunnel engineering, and elaborates the basic structure of the BIM technology implementation team from eight respects. Second, this paper elaborates the general principles and the basic process of BIM technology application in tunnel engineering. Third, the paper proposes the initial construction scheme of the tunnel engineering collaborative management platform based on BIM technology, and analyzes the feasibility of platform development deeply. Last, the BIM technology is applied to two projects including Tunnel 1 in Yinxi Railway Huanxian County and Tianjin Metro Line 6, which provides auxiliary technical means for solving practical engineering problems, and provides some reference for subsequent application researches of BIM-like technologies in tunnel engineering.
\end{abstract}

Keywords: tunnel engineering, BIM technology, principles, process, collaborative management.

\section{Introduction}

BIM (Building Information Modeling) originated in the United States in the 1970s. Charles Eastman, Jerry Laiserin and some other scholars have redefined its concepts successively and made a comprehensive interpretation of its connotation (Yalcinkaya \& Singh, 2015; Zhang, Pan, Wang, Sun, \& Wang, 2017). As a new concept and technology, BIM has been widely concerned by scholars and industry insiders in North America, Northern Europe and other areas, and has set off an unprecedented BIM technology frenzy. So far, the development of BIM in some countries such as the United States has been relatively mature, and the United States, some countries in Northern Europe, Japan, Australia, etc. have successively issued their own BIM technical standards, which has laid a solid foundation for the next development of BIM tech- nology (Jeong, 2018; Ganbat, Chong, Liao, \& Wu, 2018). BIM technology is a digital representation of all geometric and non-geometric features in construction engineering, which runs through the planning, design, construction as well as operation and maintenance phases of the project, and promotes the synergy between the project stakeholders, design institutes, construction units and monitoring units. BIM technology has five advantages, such as visualization, coordination, simulation, optimization and imageability. These advantages make it widely used in the field of construction engineering. Based on some engineering application cases, many scholars have carried out a series of related researches, which are embodied in quality monitoring (Wong, Salleh, \& Rahim, 2015), risk assessment (Ryu, Kim, S. Suh, \& W. Suh, 2015), carbon emis-

${ }^{*}$ Corresponding author. E-mail: songzhpyt@xauat.edu.cn

\#Corresponding author. E-mail: xajdwangjunbao@163.com 
sions and thermal comfort (Peng \& Wu, 2015; Marzouk \& Abdelaty, 2014), environmental protection (Liu, Meng, \& Tam, 2015; Lee, Tae, Roh, \& Kim, 2015; Marzouk, Azab, \& Metawie, 2016; Lu, Wu, Chang, \& Li, 2017; Porwal \& Hewage, 2012, 2013; Chaves, Tzortzopoulos, Formoso, \& Biotto, 2017; Reychav, Leitan, \& McHaney, 2017), energy consumption (Abanda \& Byers, 2016; Abanda, Vidalakis, Oti, \& Tah, 2015; Franeisco, Truong, Khosrowpour, Taylor, \& Mohammadi, 2018; Sanhudo et al., 2018), safety (Martinez-Aires, Lopez-Alonso, \& Martinez-Rojas, 2018; Zhang, Teizer, Lee, Eastman, \& Venugopal, 2013) and so on. On the other hand, some scholars have tried to combine BIM technology with wireless real-time sensing technology (Lai, Qiu, Chen, Wang, \& Fan, 2014; Cheung, T. H. Lin, \& Y. C. Lin, 2018; Alves, Carreira, \& Costa, 2017), 3D laser scanning technology and point cloud technology (Mahdjoubi, Moobela, \& Laing, 2013; Macher, Landes, \& Grussenmeyer, 2017) in practical engineering, based on which a lot of researches have been carried out.

With the improvement of the economy, the infrastructure construction in the world has developed rapidly, and the number of tunnels in the fields of railways, highways, water conservancy and urban underground space development has also increased, and a large number of related studies on tunnel engineering have emerged (Lai et al., 2018; Liu et al., 2019; Song, Yang, \& Jiang, 2016; Wang, Li, \& Song, 2019; Wang, Huo, Song, \& Zhang, 2019). Especially in China, in recent years, long and large, extra-long tunnels and large-scale tunnel groups have emerged. The tunnel project management has become a major problem that restricts the development of tunnel engineering. The unique advantages of BIM technology provide technical means and ways to solve this problem, making tunnel engineering integration, informatization and collaborative management possible (Song et al., 2018; Shi et al., 2017; Xiao et al., 2017). Therefore, some scholars have tried to introduce BIM technology into the field of tunnel engineering. Zhou et al. (2017) systematically analyzed the application status of BIM in China tunnel engineering through the summary and induction in terms of the lack of standards, software incompatibility and management confusion in the tunnel engineering application of BIM technology, and make some application prospect of BIM technology in tunnel engineering. Analyzing the requirements of data modeling methods and infrastructure planning projects, Borrmann et al. (2015) proposed the concept of combining multi-scale expression with BIM, and studied the geometric semantic modeling of shield tunnels. Lee, Wang, Lo, and Long (2018) proposed the system framework of BIM-3DGIS, developed corresponding maintenance management functions, and verified the applicability of the system framework. Koch, Vonthron, and Konig (2017) put forward a tunnel information modeling framework that basically integrates four interconnected subdomain models and interconnected project performance data, and based on the proposed framework, selected case studies to verify its potential and advantages.

Although BIM technology has begun to be applied in the field of tunnel engineering, since BIM technology initially originated in the field of construction engineering, its application in the field of construction engineering is more mature than the field of tunnel engineering. The reason is that the tunnel project is featured by its strip distribution, and the terrain and geological conditions are complex and changeable, which brings about the coding classification and the great differences between industry standards, software platform and the construction engineering. To realize the application of BIM technology in the field of tunnel engineering, it is necessary to establish an independent BIM technology application system suitable for tunnel engineering. On the other hand, BIM technology has the characteristics of granularity. Component is the minimum granularity for information transmission and sharing. Therefore, as the basic element of BIM technology, component is very important for a project. Taking Revit software as an example, most of the family components in Revit software are only suitable for the field of construction engineering, and there are few family components in the field of tunnel engineering. This leads to the lack of component libraries in the tunnel engineering field, which directly or indirectly causes many difficulties and bottlenecks in the tunnel engineering foundation modeling process.

In summary, the development of BIM technology in the field of tunnel engineering still has certain limitations, which are embodied in the inconsistency of standards, the inapplicability of software, and the imperfection of platforms. Therefore, it is very important to carry out relevant researches on the standardized application of BIM technology in the field of tunnel engineering. The research results are conducive to the development and maturity of BIM technology in the field of tunnel engineering. Thus, the article elaborates the key links involved in the application of BIM technology in tunnel engineering from the aspects of hardware and software configuration requirements, implementation team organization structure, BIM technology implementation principles, BIM technology implementation process and tunnel collaborative management platform based on BIM technology, providing some reference for the standardized application of BIM technology in the subsequent tunnel engineering field. The BIM technology application system and the initial framework of the tunnel collaborative management platform in tunnel engineering proposed in this paper are all proposed for the first time. They have certain integrity and systematics, and are highly implementable, which is also a relatively novel and unique point in this paper. 


\section{Application fundamentals and process of BIM technology in tunneling}

\subsection{Software and hardware conditions for BIM technology application}

\section{Software conditions for BIM technology application}

At present, BIM series software mainly includes core modeling software, deepening design software, structural analysis software, collision checking software, sustainable analysis software, cost management software and operation management software. Each software series contains countless kinds of various software. The BIM core modeling software is divided into four series: Autodesk, Bentley, Nemetschek Graphisoft, and Dassault. Figure 1 shows the hardware and software conditions for BIM technology application diagram. Among the four series, Autodesk's Revit architectural, structural, and electromechanical series are most widely used, and Bentley's architectural, structural, and equipment series are mainly used in industrial design and municipal infrastructure. However, products in Nemetschek Graphisoft and Dassault are seldomly used.

In order for BIM technology to be successfully applied in engineering projects, the first step is to develop a reasonable software selection plan. It is well known that many of the current software are developed based on the background of construction engineering, and its application in tunnel engineering will inevitably have certain limitations, affecting the application of BIM technology in tunnel engineering. Considering the characteristics of tunnel engineering, after software optimization, the software scheme of tunnel engineering BIM technology implementation in this paper is as follows: BIM technology software is mainly based on Autodesk series software, supplemented by some other series of software. In view of the current lack of proprietary software series in the field of tunnel engineering, the BIM software series in the tunnel engineering field will be gradually developed in the later stage to better serve the actual engineering projects.

\section{Hardware conditions for BIM technology application}

Hardware and software are two independent parts of a complete computer system. After we have determined the BIM software to use, we need to consider how the hardware should be configured. The working mode of BIM based on the 3D model puts high demands on the computing power and graphics processing capability of the hardware. Taking the most basic project modeling as an example, BIM modeling software, compared with the traditional two-dimensional CAD software, needs great attention in the configuration of CPU, memory, graphics card and hard disk, as is shown in Figure 1.

If the software configuration requirements are considered separately, the higher the hardware configuration standard is, the smoother the software will run and the higher the work efficiency will be. However, for the application of BIM technology in tunnel engineering, it is necessary to consider the application of BIM technology. At this time, it is not only the factors like configuration requirements of individual software products, but also the size and complexity of the project, the application target of BIM technology, and the degree of team application and

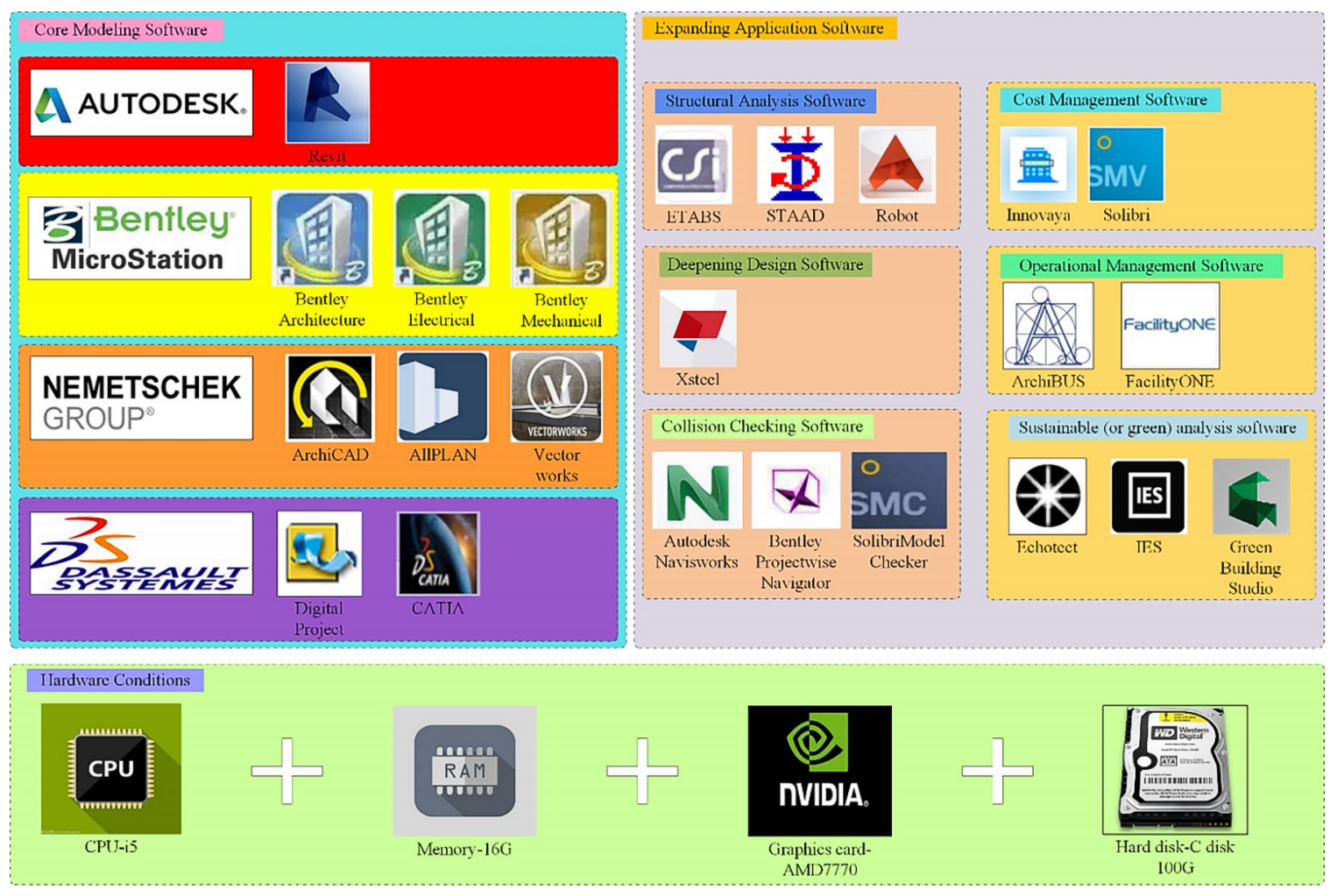

Figure 1. Diagram of hardware and software conditions for BIM technology application 
working methods that need consideration. A multi-factor comprehensive analysis can help to obtain a reasonable hardware configuration.

Based on this, the overall scheme of BIM technology hardware configuration is as follows. According to the work content of each member, different hardware is equipped to form a stepped configuration. For example, lower configurations can be considered for a single-professional modeling, while integration to specialized models requires higher configurations. Some large data volumes of analog analysis may require higher configurations. If the network cooperative working mode is adopted, the central storage and transportation office server needs to be set up. If allowed, the computer hardware configuration needs to be slightly higher than the minimum value, which can ensure the economical and stable operation of the software.

\subsection{Organization structure of BIM technology application}

As an indispensable part of the application system of BIM technology, the construction of the organization structure of BIM technology application must be explained in detail. In tunnel engineering, these organization structures also have the unique characteristics of tunnel engineering. For example, the purpose of the data collection department is to conduct monitoring and measurement, advanced geological prediction and other related data collection work; the model design department adds topographic and geological models besides the main and subsidiary models of the tunnel, which are also the difference between the organization structure in the field of tunnel engineering and that in the field of construction engineering. It is just because of the uniqueness that it is necessary to explain and analyze it here.

The application of BIM technology in the project is not completed by an individual. Instead, it requires a coordinative team where members perform their duties, so that BIM technology can be successfully applied to actual engineering projects, so as to better serve actual engineering. Therefore, in order to better implement the BIM information management model in the project, a special BIM technical management team is required. The team has one BIM technical director and two deputy directors. There are eight departments, and each deputy director is in charge of four departments. The eight departments are model design department, construction simulation department, program optimization department, data collection department, hardware maintenance department, software development department, results delivery department and operation and maintenance management department. The eight departments have their clear division of labor; they work together and manage efficiently. Figure 2 shows the organization structure of BIM technology.

\subsection{Implementation principles of BIM technology application}

The implementation of BIM technology must follow certain general principles. The establishment of the overall principle is of great significance for the preparation of

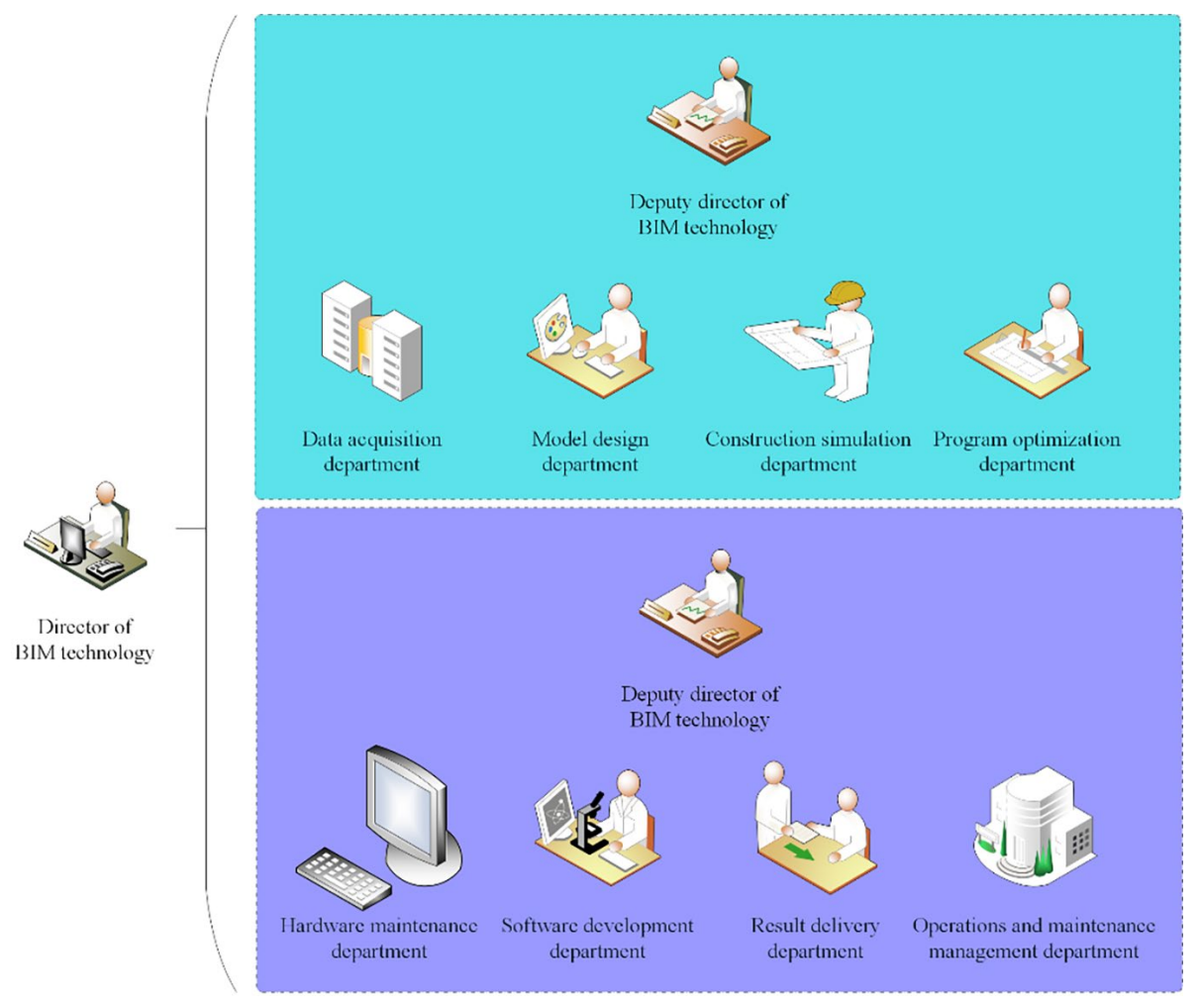

Figure 2. Organization structure diagram of BIM technology 
subsequent BIM standards in tunnel engineering and the evaluation of BIM technology results formed in the later period. As is known to all, the ultimate goal of BIM technology in tunnel engineering is to realize the information and integrated management of tunnel engineering and achieve the goal of lean management of tunnel engineering projects. The premise of information management is to ensure the authenticity and integrity of the information. For integrated management, the premise is to ensure information management coordination. According to the application characteristics of BIM technology parametric modeling and information management, the application of BIM technology is divided into three stages: early stage, middle stage and later stage. The early stage is the overall modeling stage of tunnel engineering, which needs to ensure the authenticity of information; the middle stage is the construction phase of the tunnel projects, and the integrity of the information is required at this stage; in the later stage, the collaborative management platform is built. At this stage, the collaborative management of information needs to be guaranteed. Therefore, based on the characteristics of BIM technology and the particularity of tunnel engineering, aiming at the three stages of BIM technology application, this paper puts forward three general principles for BIM technology implementation, such as information authenticity, information integrity and information management coordination:

1) Early stage - Information authenticity. The pre-implementation of BIM technology, especially the authenticity of data information in the modeling process, from terrain relief, tunnel length, length of surrounding rock sections of the tunnel, to initial support thickness, secondary lining thickness, pipe shed diameter, length and so on should all be based on actual conditions and not be fabricated at will, so that the created model can reflect the actual situation of the project and thus better serve the actual project.

2) Middle stage - Information integrity. In the mid-term of BIM technology implementation, the massive engineering data information involved in the project should be kept as far as possible, such as the change information in the project, the number of changes, the content of the changes, and the reasons for the changes from the start to the completion of the project. If all of this information is attached to the model without any omissions, a relatively complete tunnel engineering information model will be made.

3) Later stage - Information management coordination. In the later stage of BIM technology implementation, the tunnel engineering and BIM management platform has been initially formed, and certain collaborative work could be carried out. At this stage, we should pay attention to the synergy of project management, in order to maximize the construction benefits and minimize the loss in the tunnel engineering, so as to achieve the purpose of management optimization. Each participant of the project can log in to the platform and use its own authority to access the data information to grasp the progress of the project in real time, timely formulate reasonable treatment plans and collaborative management for the emergency situation to make the project more efficient.

\subsection{Implementation process of BIM technology application}

With its unique characteristics, tunnel engineering is different from housing construction. Therefore, the application points of BIM technology will naturally differ, including tunnel component library establishment, 3D tunnel model creation, 3D surface model creation, 3D geological model creation, construction technology simulation, secondary deepening design, cost control, quality control, safety control, material tracking, and virtual roaming. The implementation process of tunnel engineering BIM technology is as follows:

1) Creation of tunnel body model. BIM modeling software such as Revit is used to establish the exclusive component library of Huanxian County No. 1 Tunnel, including initial support, second lining arch wall, second lining inverted arch, inverted arch filling, steel arch, radial anchor and so on. Then, based on the existing exclusive tunnel component library, the hole section, the hole body and the auxiliary cavern are respectively established, and a complete tunnel engineering civil construction model and structural model are established. In addition, the accuracy control established by the model needs to be determined according to the requirements of the later application.

2) Creation of $3 \mathrm{D}$ topographic geological model. The tunnel project is a strip-shaped distribution, and the span is often large. The change of geological conditions will change the original excavation plan of the tunnel, which in turn adapt to the new geological environment. The topographical geological conditions around the tunnel function as a more important part of the tunnel engineering. Therefore, it is necessary to model the topographic geology to form a 3D terrain model and a 3D geological model. These two models can also be created by modeling software such as Revit. If possible, the terrain model can be obtained by UAV scanning, which is more accurate.

3) Collision inspection and deepening design. For underground projects such as subway projects and integrated pipe corridors, necessary pipeline collision inspection and deepening design are required. For the mountain tunnel project, the pipeline distribution is less, and collision inspection can be omitted. However, in order to meet the depth requirements of later applications, it is necessary to complete the deepening design of some key nodes, including the complex points of steel bar binding, steel frame connection parts, advanced support positions and special construction techniques.

4) Simulation of construction process and schedule. Using Navisworks, Guanglianda BIM5D and other relevant construction simulation software to carry out the 
necessary construction process simulation, combined with the Midas numerical calculation model, can reoptimized the original construction process can be to avoid rework, which shows great significance to the process control and cost savings.

5) Cost management. Through the secondary development in the later stage, a tunnel engineering collaborative management platform is formed. After the model and model-based information and data are uploaded to the platform, based on the platform, the real-time engineering quantity of the tunnel engineering can be extracted according to the construction progress. And based on the real-time engineering quantity, the realtime cost of the tunnel engineering can be generated. The participants of the project can use the platform to conduct real-time query, extraction and management of real-time costs.

6) Quality and safety management. Real-time monitoring of material quality, construction quality and construction safety is made through new technologies such as remote data transmission and Internet of Things to ensure that quality meets standards and that safety work is in place.

\section{Design and development of the collaborative management platform}

\subsection{Core development technology}

The core development technologies of the platform include BIM technology, GIS technology, Internet of Things technology and cloud storage technology. BIM technol- ogy is responsible for classifying and managing the tunnel body information model imported into the platform. GIS technology is responsible for classifying and managing the digital ground model and 3D geological model imported into the platform. The Internet of Things technology is responsible for the information of personnel and materials in the tunnel project. In addition, the platform involves the storage of massive data information, using distributed cloud computing technology, and cloud storage technology to solve the storage problem of massive data information. These data information is stored to form a central database, which is managed by a central server. The central server classifies the data into professional databases and each of them is responsible for a branch professional database. Each professional database is under the support of BIM technology, GIS technology and Internet of Things technology, forming various professional functional modules to create a platform end. The user and the platform ends realize human-computer interaction through the network. The user accesses the platform through the network to obtain data information related to the tunnel project. Figure 3 shows the core technology of the tunnel engineering collaborative management platform.

\subsection{Functional module design}

The paper designs the tunnel collaborative management platform into eight modules from the functional aspect. The eight modules are tunnel component management module, tunnel model management module, tunnel terrain management module, tunnel schedule management module, tunnel cost management module, tunnel quality

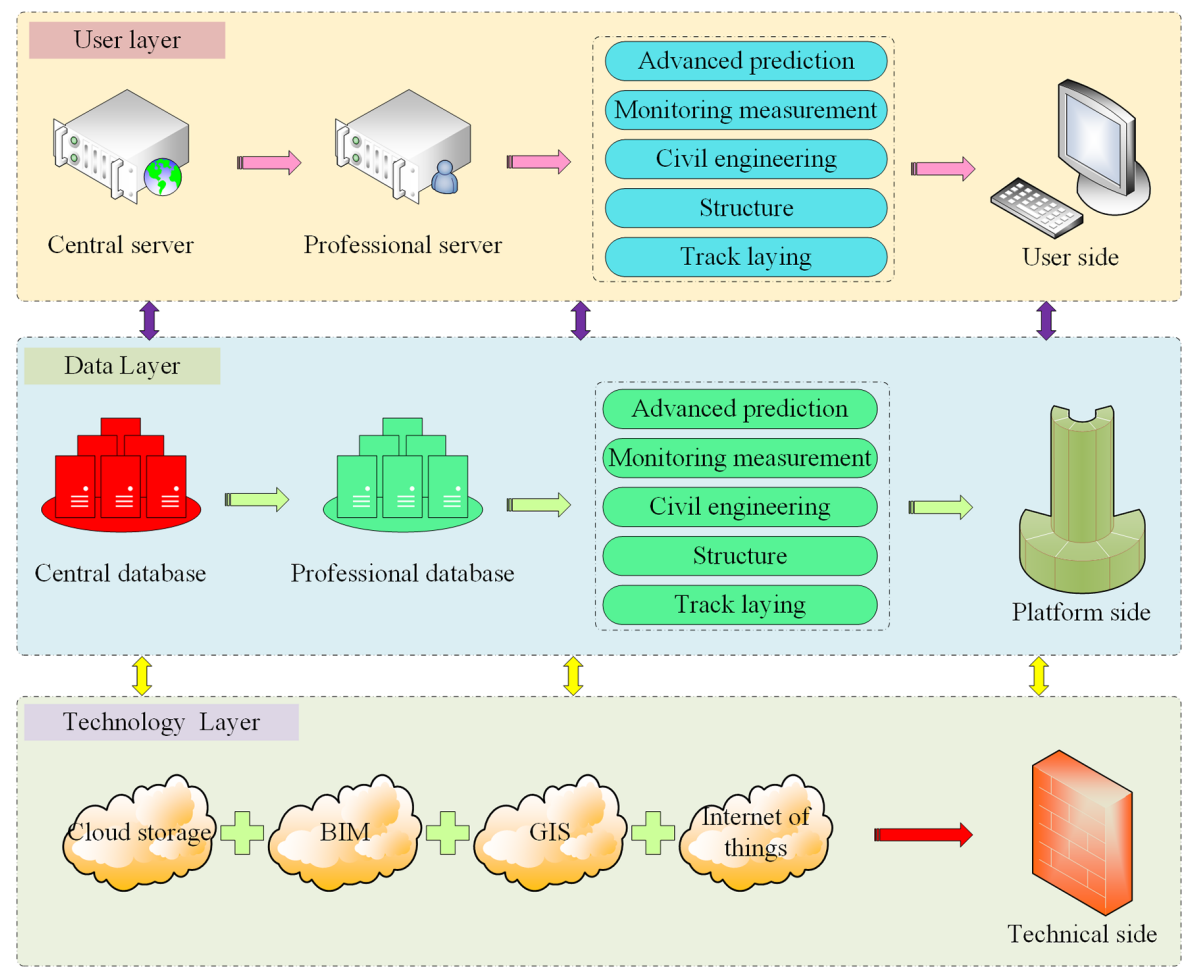

Figure 3. Core technology diagram of tunnel engineering collaborative management platform 
management module, tunnel energy management module and tunnel security management module:

1) Tunnel component management module design. Presently, most BIM technology software is developed on the basis of construction engineering. The components attached to Revit software, for instance, belong to the field of construction engineering, and there are few exclusive components in the field of tunnel engineering. Therefore, for the application of Revit series software in tunnel engineering, the premise of establishing a complete tunnel model is to create some special components in the tunnel engineering fields such as initial support, secondary lining, inverted arch, steel frame and steel bar. A dedicated component library gradually formed by accumulation in the field of tunnel engineering can be used twice in the later tunnel engineering modeling. The tunnel component library management module can realize the function of classification and management in the component library. The module supports the import, upload and download of the exclusive components of tunnel engineering, and can export the two-dimensional plan paper marked with the detailed dimensions of the exclusive components. In addition, the module can query and export the component attribute information, and can dynamically update the component library at any time to realize the dynamic management of the tunnel engineering component library.

2) Tunnel model management module design. The object of the tunnel component library management module is the component, and the object of the tunnel model management module is the entire tunnel model. The module can automatically save the tunnel model imported to the platform each time, and gradually accumulates a model library through long-term management of different projects. The module supports the import, upload and download of the tunnel engineering model. The user can call the tunnel model of the model library at any time, and browse the tunnel model in real time based on the 3D visualization interface of the platform. On the other hand, in the design and construction stage, problems can be found in time based on this module. The model can be changed accordingly and the changed model can be uploaded to the platform. The module can automatically identify the change status of the model, and all participants in the project can grasp the change information at the first time and take the following measures in time. In addition, the module can export the two-dimensional plan paper based on the $3 \mathrm{D}$ model to realize standardized and rapid drawing of tunnel engineering.

3) Module design for tunnel terrain management. The tunnel project has the characteristics of strip distribution, and its spatial span is large, which leads to the complicated and varied geological conditions of the areas it passes through. Before the tunnel design, it is necessary to know and grasp some specific information about the location of the tunnel in advance, such as whether there are any geological phenomena such as folds and faults in the tunnel crossing area, whether the stratum is the soil layer or the rock layer, what grade the surrounding rock is, and the hydrogeological situation of the tunnel crossing area. Such information is directly related to the planning and design of the entire tunnel project. Therefore, understanding and mastering the terrain and geological conditions are very important for tunnel engineering. The tunnel terrain management module is a functional module based on this. The module supports the digital ground model based on GIS technology and the import, upload and download of 3D geological models based on geological 3D reconstruction technology, and can realize the integration of 3D terrain model, 3D geological model and 3D tunnel model. The platform performs real-time browsing based on 3D visualization and can query the relevant terrain and geological information required based on the model. In addition, the uploading and management of related satellite and aerial image data of the tunnel project location area can be realized. The user can query and call at any time according to their demand.

4) Module design for tunnel schedule management. In the tunnel schedule management module, the record of the construction schedule and the actual construction progress information in real time with the help of the $3 \mathrm{D}$ visualization model can help the user clearly grasp the construction dynamics and ensure that the whole project is completed on schedule; in addition, the tunnel construction method library is entered, so the tunnel construction technology such as the step method, the three-step method, the six-step CD method and the middle partition method can be inquired in the module. Each construction technology has a corresponding construction simulation video in addition to the related text description, which can better guide the actual construction. On the other hand, the digital device collects the construction parameters to form the construction log. The electronic construction log and the report generated by the professional software data analysis are uploaded to the platform. The module can classify and manage the electronic data, and the participants of the project can has a more comprehensive grasp of the construction process based on this module.

5) Module design for tunnel costs management. The tunnel cost management module can be divided into two parts, one of which is the calculation function, that is, the engineering quantity statistics and inventory management of the whole tunnel project; the other is the cost function, that is, for a certain construction scheme, the engineering cost is carried out basis of the calculation. For a tunnel project, the best cost-effective project is the best solution. The proposing and post-development of the module can help reduce project cost and optimize the design of the project.

6) Module design for tunnel quality management. The tunnel quality management module supports the management of the tunnel construction quality, the quality 
of the materials used and the quality of the prefabricated components. It can also query the construction technology standards, material standard indicators and prefabricated component design specifications. In addition, the field construction technology, materials used, prefabricated components, and some other actual information can be uploaded to the platform, and the module can finally give an evaluation report of the construction quality through comparative analysis. It can be seen that this module has important significance in quality control.

7) Module design for tunnel energy management. The module follows the concept of sustainable development, and monitors and manages the energy consumption during tunnel operation and maintenance. According to the energy consumption source in the tunnel engineering operation management, the module is now divided into tunnel lighting energy consumption analysis and tunnel ventilation energy consumption analysis. The module can make relevant energy analysis of the model and give an energy analysis report based on the model imported to the platform, and then according to the report develops or adjusts the operation plan to achieve green management of the tunnel project.

8) Module design for tunnel safety management. Based on the concept of the whole life cycle of tunnel engineering, the tunnel safety management module can be divided into construction safety management and operational safety management. In terms of construction safety management, the platform can realize real-time uploading of monitoring data such as tunnel arch settlement and surrounding rock deformation. The module can make intelligent judge and alarm based on uploaded monitoring data to ensure construction safety. In terms of operational safety management, the module supports real-time monitoring and automatic alarm of tunnel fires. In addition, the module can analyze the monitoring data such as crack monitoring and side wall water seepage monitoring in tunnel maintenance, and timely alarm to realize tunnel operation safety.

\subsection{Feasibility analysis}

Feasibility analysis levels are as follows:

1) Technical level: the proposed technology for tunnel engineering collaborative management platform development is BIM technology, GIS technology, cloud storage technology and Internet of Things technology. Most of these technologies are very mature, and have a solid technical foundation and strong development operability.

2) Functional level: the functional design of the platform is based on the unique characteristics of the tunnel engineering and meets the actual needs of the tunnel engineering field. In addition, the functional design also combines the application concept of the whole life cycle of BIM technology. With the joint assistance of the tunnel engineering planning, design, construction and operation and maintenance stages, the application value of tunnel engineering BIM technology can be maximized.

3) Application level: tunnel engineering is strip engineering; the space span is large, and the geological conditions along the line are complex and changeable. The development of the platform is in line with the development trend of efficient construction and collaborative management of tunnel engineering, and conforms to the development policy of the new era of the country. Therefore, it is quite necessary to develop the platform.

\section{Engineering application cases}

\subsection{Tunnel 1 in Huanxian County}

Tunnel 1 in Huanxian County is one of the key projects of the newly built Yinxi Railway, which is located in the three provinces (regions) of Shaanxi, Gansu and Ningxia Autonomous Region, connecting the Guantian Economic Zone, the Jidong Region and the Huangcheng City Belt. The line starts south from Xian, the central city of the northwest region, and goes to the northwest of Xianyang City, Shaanxi Province, Qingyang City, Gansu Province, and Wuzhong City, Ningxia Autonomous Region, and then enters Yinchuan City, the capital of Ningxia Autonomous Region. The total length of the railway is $616.81 \mathrm{~km}$.

Tunnel 1 in Huanxian County is located in Dongshan, Huanxian County, Gansu Province. The area that the tunnel passes through is located in the loess wreckage area on the east bank of the Huanjiang River. The terrain is undulating and the ground elevation is $1250-1440 \mathrm{~m}$. The starting point of the tunnel is DK370+386.31, the ending mileage is DK374+717.655, and the total length is $4331.345 \mathrm{~m}$. It is a single-hole double-line tunnel. Figure 4 shows the location of the Tunnel 1 in Huanxian County.

At present, the two-dimensional drawings are still used in most tunnel projects, and Tunnel 1 in Huanxian County is no exception. Because of the limitation of twodimensional planarization of drawings, there are often some problems such as mistakes, leaks and bumps. If these problems are not found and corrected in time, they will directly affect the construction in the later stage, resulting in the extension of construction period and the increase of project cost. Secondly, Tunnel 1 in Huanxian County belongs to a long-distance tunnel with a large regional span and complicated and changeable geological conditions along the tunnel. The location and alignment design of the tunnel are very important. The design of two-dimensional drawings, due to the limitation of space perspective, is also prone to the problems of pre-planning, which is extremely disadvantageous to the design and construction of the latter. In addition, Tunnel 1 in Huanxian County belongs to large cross-section loess tunnel, which requires a high degree of convergence of construction technology and construction sequences. It is difficult to meet the new requirements of tunnel construction in the new era by 
relying solely on relevant instructions and two-dimensional drawings design, and new technology needs to be introduced as a new solution. With this, this paper puts forward a solution based on BIM technology and applies it to Tunnel 1 in Huanxian County; Figure 5 shows the application of BIM technology in Tunnel 1 in Huanxian County. The specific schemes are as follows:

1) Solution in the planning stage: the establishment of terrain and geological model, the creation of terrain model with Civil 3D and 3ds Max, the creation of geological model with Revit, and the screening and optimization of tunnel engineering planning scheme based on the established terrain model and geological model are applied.

2) Solution in design stage: Autodesk series software Revit is used to create the main model of tunnel. Based on the built tunnel model, the errors of two-dimensional drawings are checked, especially the design of steel bar and steel frame. Based on the characteristics of BIM technology such as information and three-dimensional visualization, the errors of existing two-dimensional design are checked and corrected in time.

3) Solution in construction stage: Navisworks software is used to carry out relevant simulation to ensure the coordination and orderliness of each step of tunnel construction and visualization of key processes, reducing the difficulty of construction and ensuring the safety of construction.

\subsection{Tianjin Metro Line 6}

The starting point of the section of Heiniucheng Road Station to Meijiang Road Station of Tianjin Metro Line 6 is located at Heiniucheng Road Station on Jianshan Road, and the end point is at Meijiang Road Station on Embankment Road 5. From the north to the south, the section passes through the Black Bull Road Expressway,

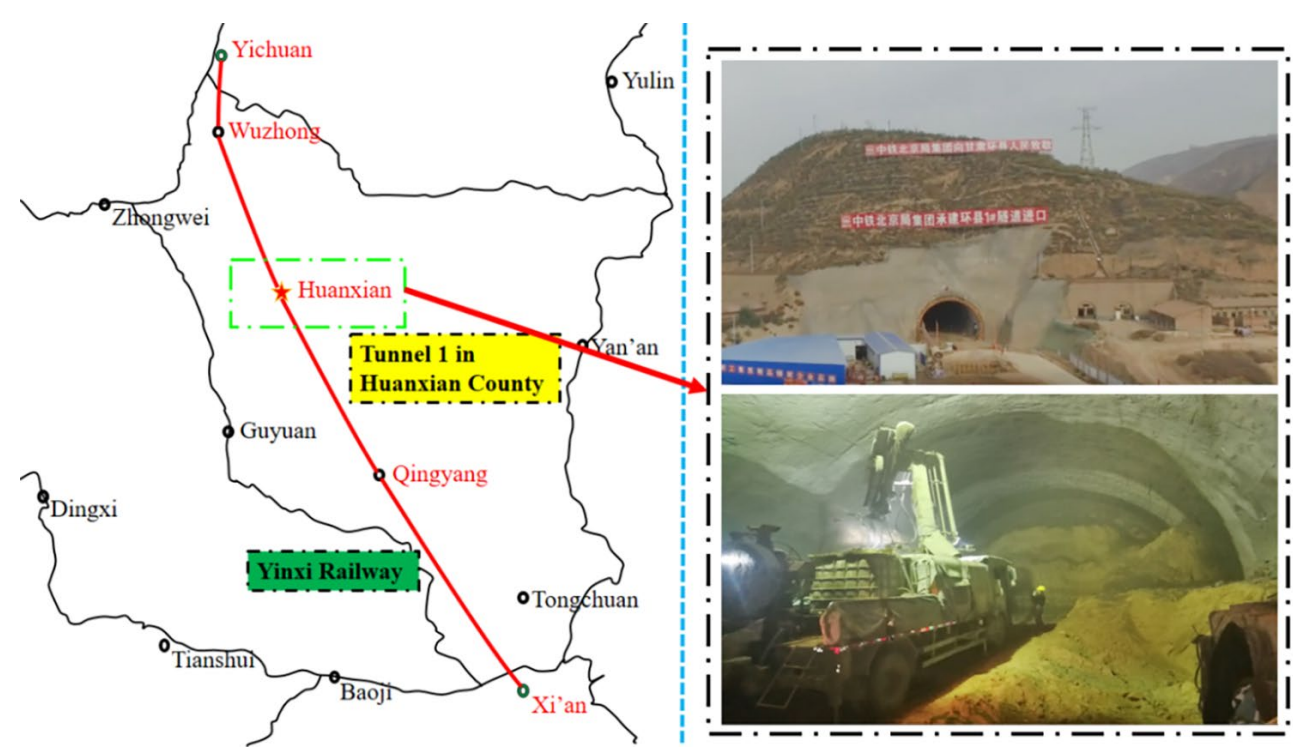

Figure 4. Diagram of the location of Tunnel 1 in Huanxian County

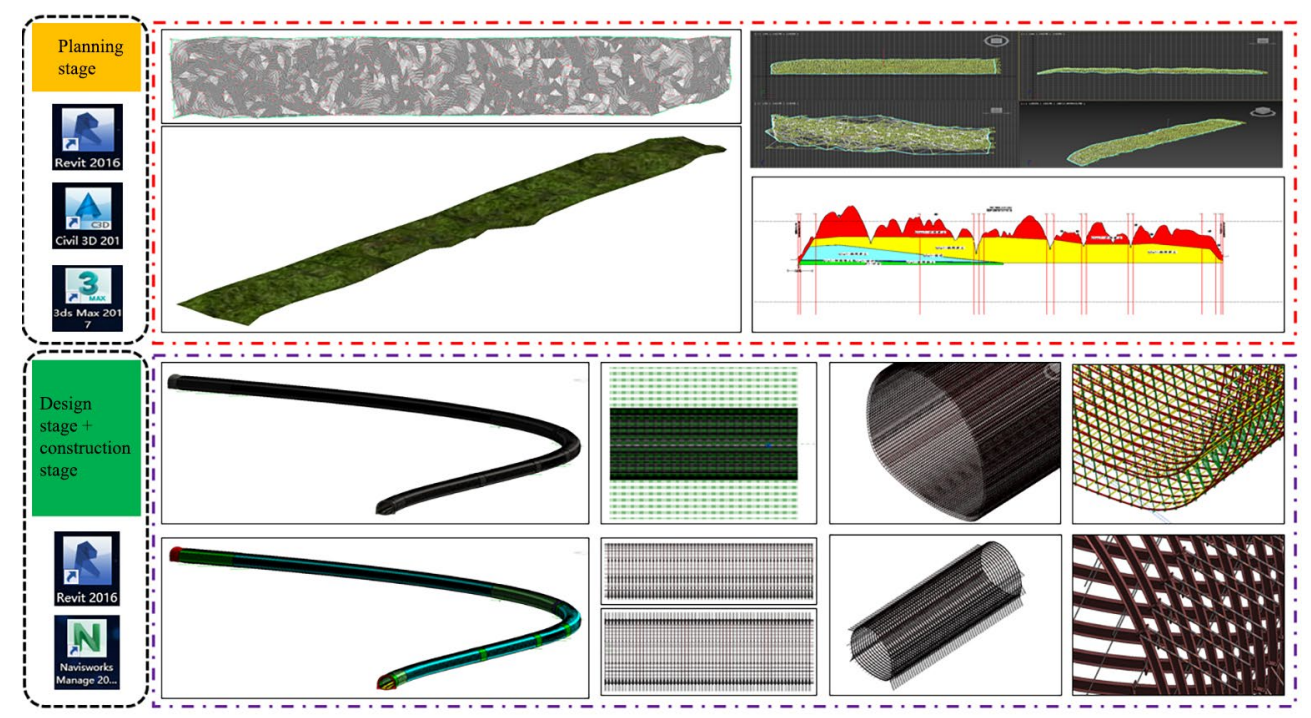

Figure 5. Diagram of BIM technology application in Tunnel 1 of Huanxian County 
the Chentangzhuang Railway Freight Station and the Fuxing River. The starting and ending mileage of the interval is DK33+556.074 DK34+749.205, the right line length is $1192.814 \mathrm{~m}$, the left line length is $1192.381 \mathrm{~m}$, and a communication channel and pump room is set at the mileage DK34+153.355. The receiving end of the section shield is the Heiniucheng Road Station, which is located at the intersection of Heiniucheng Road and Jianshan Road in Hexi District. It is arranged in the north-south direction along Jianshan Road. The station is a two-layer, three-span island station on the underground, with a part of threelayer underground transfer. Figure 6 shows a diagram of the section of Heiniucheng Road Station to Meijiang Road Station of Tianjin Metro Line 6.

Municipal pipelines are distributed at the receiving end of shield machine. In order to ensure the safety of underground pipelines and upper buildings at the receiving end, no errors are allowed in the process of shield machine receiving. The biggest risk point of the whole steel sleeve receiving scheme lies in the control of the installation axis of the steel sleeve and the shield axis, which does not allow too large deviation of the axis. In addition, besides shield construction, there are also construction steps of soil reinforcement, steel sleeve installation and shield passing through the reinforcement area. It is difficult to ensure the perfect connection of construction steps only by two-dimensional drawings and related construction organization design. New technology is needed to simulate the whole receiving scheme. Based on which, this paper proposes a solution based on BIM technology to assist the smooth implementation of shield receiving scheme. Figure 7 shows a diagram of the simulation of the shield receiving scheme of Tianjin Metro Line 6. The specific solutions are as follows:

1) Solution in scheme design stage: The model of steel sleeve receiving scheme is established by using Autodesk series software Revit, and then the design dimension of steel sleeve is checked based on the model. Whether the steel sleeve is suitable for the installation of hoisting shaft and whether the location of the installation axis of the steel sleeve is consistent with that of the shield tunnel should be adjusted in time at this stage if there are any problems.

2) Solution in the plan implementation stage: Navisworks software is used to simulate the whole shield receiving scheme. Several key construction steps are simulated, such as soil reinforcement, steel sleeve installation, shield passing through reinforcement area, shield receiving completion, steel sleeve demolition, etc. The problems in connection of construction steps are adjusted in time, and the adjusted final model guides the implementation of the actual scheme to ensure the smooth completion of shield receiving work.

\subsection{Case analysis}

In the above two engineering cases, BIM technology has been applied to some extent. In the aspect of planning and design, the overall optimization of the planning scheme was carried out, which reduced the design error rate. In the aspect of construction, through simulation, the complicated construction sequences could be adjusted and optimized, which could better guide the actual construction. As an auxiliary technology, BIM technology has solved some problems in practical engineering and achieved certain economic and social benefits. On the other hand, the application scope of BIM technology should eventually cover the whole life cycle of tunnel engineering and realize the integrated information management of the whole life cycle of tunnel engineering, which is also the core essence of BIM technology. At present, the two engineering cases involved in this paper are limited to the planning, design and construction stages, but this is the first step in the application of BIM technology in tunnel engineering. The application of BIM technology in the later stage of operation and maintenance needs to be realized after the completion of the development of tunnel cooperative management platform, which is the limitation of this study and the future direction of in-depth research.

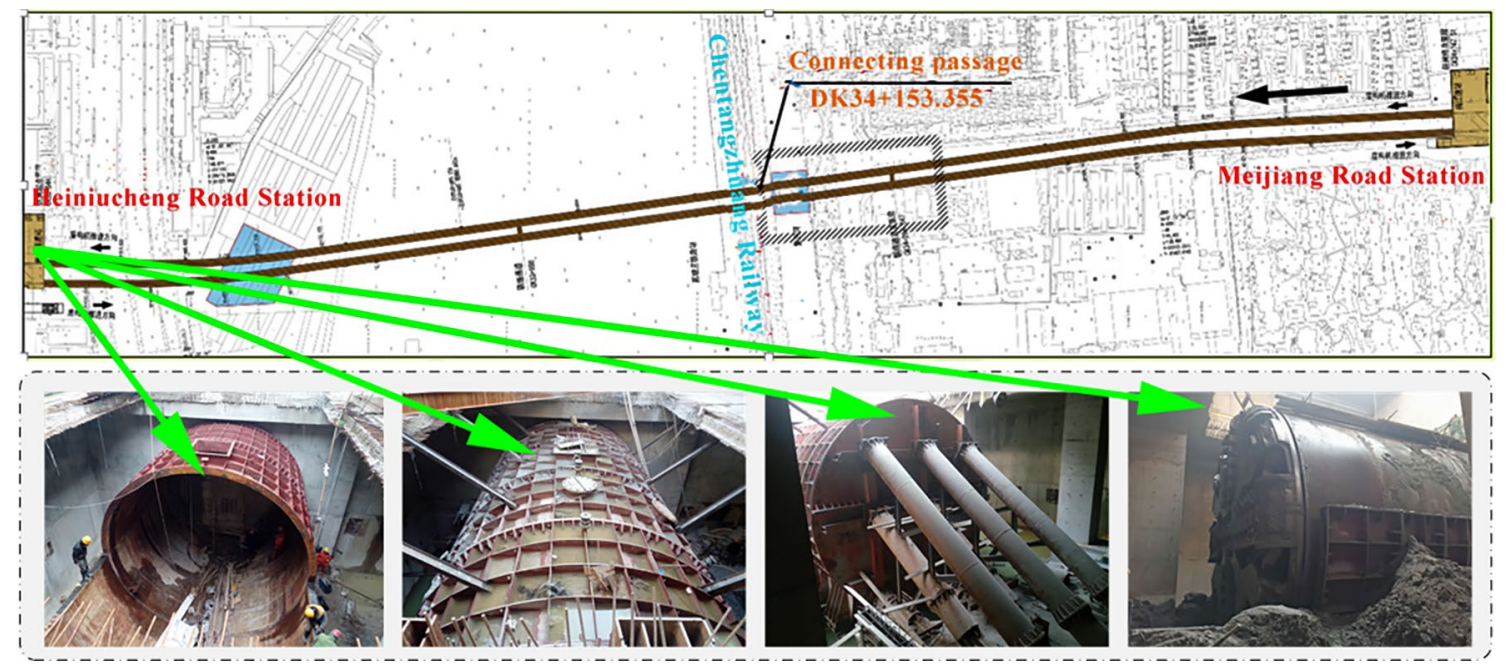

Figure 6. Diagram of the section of Heiniucheng Road Station to Meijiang Road Station of Tianjin Metro Line 6 


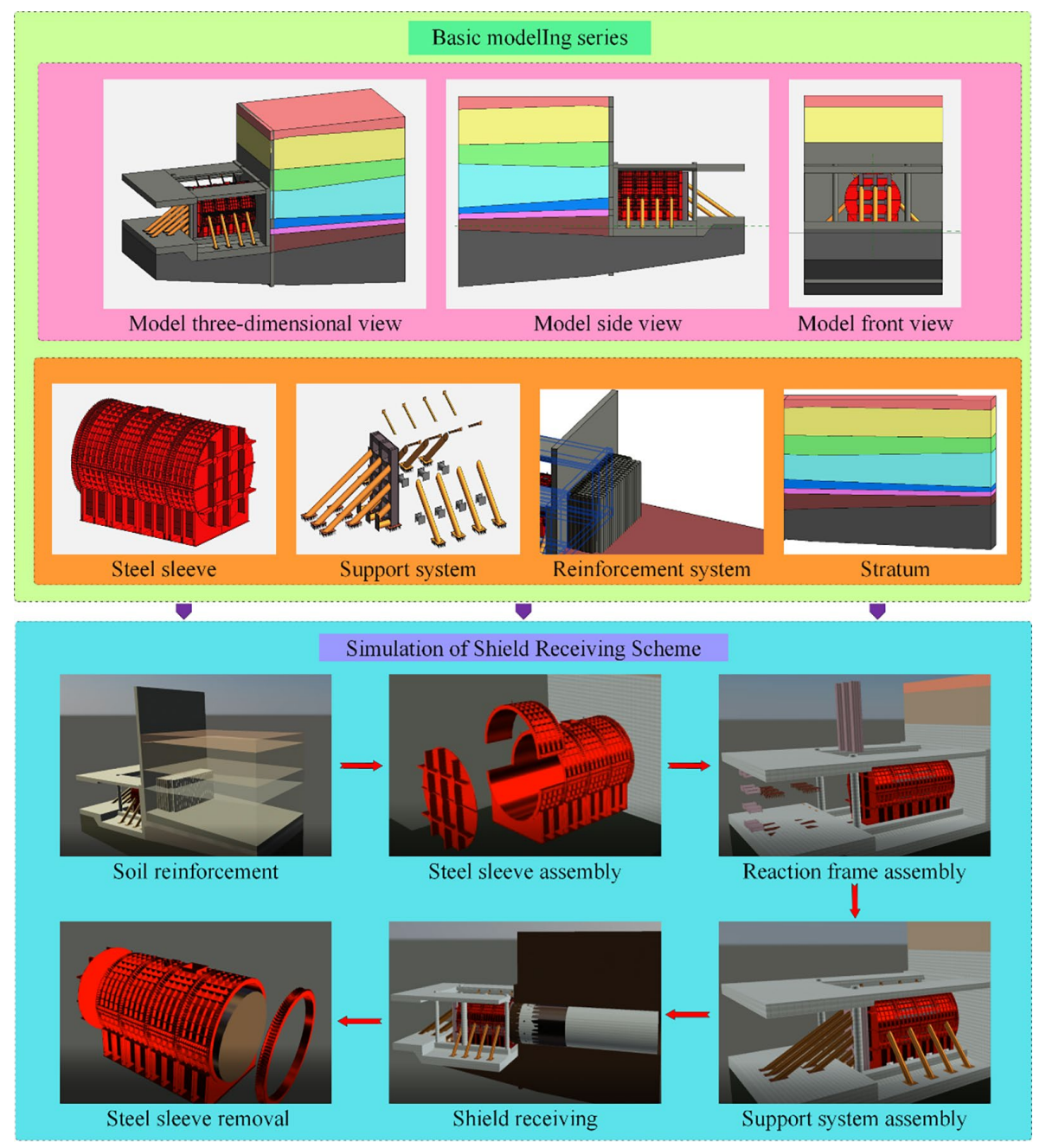

Figure 7. Simulation of shield receiving scheme for Tianjin Metro Line 6

\section{Conclusions}

Tunnel engineering contains a large amount of engineering data and information. Informatization and digitization will become an important topic in the future development of tunnel engineering, and BIM technology will become an important technical foundation for realizing this theme. Therefore, it is of great significance to carry out related research on the application of BIM technology in tunnel engineering. BIM technology originated in the field of construction engineering, and its application in the field of construction engineering is relatively mature. However, the development of BIM technology in tunnel engineering is still in its infancy. Based on this, this paper established a complete application system of BIM technology in the field of tunnel engineering, as well as a preliminary framework of tunnel collaborative management platform, which lays a good foundation for realizing the collaborative management of BIM technology in the field of tunnel engineering. In addition, "one system and one platform" in this paper is proposed for the first time in terms of integrity and systematics, which is also the highlight of this paper. The research work of this paper are summarized as follows:
1) The paper puts forward the basic hardware and software configuration requirements for BIM technology application, makes comparison and analysis, and gives the software and hardware solutions commonly used in implementing BIM technology in tunnel engineering. In addition, the article sets up the basic sections of BIM technical team in eight aspects of model design, construction simulation, program optimization, data acquisition, hardware maintenance, software development, results delivery and operation and maintenance management. The eight departments work together to constitute the entire BIM technology implementation team.

2) The paper puts forward the general principle of BIM technology application in tunnel engineering, and introduces the basic connotation of several principles from three aspects: information authenticity, information integrity and information management synergy. On the other hand, on the basis of the overall principle, the paper puts forward the basic process of BIM technology application, and makes a detailed explanation of each item in the process. 
3) This paper proposes the initial organization scheme of the tunnel engineering collaborative management platform based on BIM technology, and carries on the preliminary design and division of the main functions of the platform. Additionally, the paper discusses the feasibility of platform development from the technical level, functional level and application level to make more detailed analysis. In engineering application, the paper applies BIM technology to two actual projects, such as Tunnel 1 in Huanxian County of Yinxi Railway and Tianjin Metro Line 6, which provide auxiliary technical means for solving practical engineering problems.

Besides, it should be noted that the collaborative management platform proposed in the article is only a preliminary organization plan, and the platform development work is still in progress. However, the technical foundation of the current platform development has been relatively mature, and the development feasibility is high. In the follow-up study, the development of the platform will continue, and further research will be conducted based on this.

\section{Acknowledgements}

This paper is supported by the National Natural Science Foundation of China (No. 51578447), the Youth Science and Technology Nova Program of Shaanxi Province (No.2018KJXX-061), and the Technology Research and Development Project Foundation by the Ministry of Housing and Urban-Rural Development of China (No. 2017-K4-032). The financial supports are gratefully acknowledged by the authors.

\section{References}

Abanda, F. H., \& Byers, L. (2016). An investigation of the impact of building orientation on energy consumption in a domestic building using emerging BIM (Building Information Modelling). Energy, 97, 517-527.

https://doi.org/10.1016/j.energy.2015.12.135

Abanda, F. H., Vidalakis, C., Oti, A. H., \& Tah, J. H. M. (2015). A critical analysis of Building Information Modelling systems used in construction projects. Advances in Engineering Software, 90, 183-201.

https://doi.org/10.1016/j.advengsoft.2015.08.009

Alves, M., Carreira, P., \& Costa, A. A. (2017). BIMSL: A generic approach to the integration of building information models with real-time sensor data. Automation in Construction, 84, 304-314. https://doi.org/10.1016/j.autcon.2017.09.005

Borrmann, A., Kolbe, T. H., Donaubauer, A., Steuer, H., Jubierre, J. R., \& Flurl, M. (2015). Multi-scale geometric-semantic modeling of shield tunnels for GIS and BIM applications. Computer-Aided Civil and Infrastructure Engineering, 30, 263-281. https://doi.org/10.1111/mice. 12090

Chaves, F. J., Tzortzopoulos, P., Formoso, C. T., \& Biotto, C. N. (2017). Building information modelling to cut disruption in housing retrofit. Proceedings of the Institution of Civil Engineers-Engineering Sustainability, 170, 322-333.

https://doi.org/10.1680/jensu.16.00063
Cheung, W. F., Lin, T. H., \& Lin, Y. C. (2018). A real-time construction safety monitoring system for hazardous gas integrating wireless sensor network and building information modeling technologies. Sensors, 18(2), 436.

https://doi.org/10.3390/s18020436

Franeisco, A., Truong, H., Khosrowpour, A., Taylor, J. E., \& Mohammadi, N. (2018). Occupant perceptions of building information model-based energy visualizations in eco-feedback systems. Applied Energy, 221, 220-228. https://doi.org/10.1016/j.apenergy.2018.03.132

Ganbat, T., Chong, H. Y., Liao, P. C., \& Wu, Y. D. (2018). A bibliometric review on risk management and building information modeling for international construction. Advances in Civil Engineering. Article ID 8351679.

https://doi.org/10.1155/2018/8351679

Jeong, Y. (2018). A study on the BIM evaluation, analytics, and prediction (EAP) framework and platform in linked building ontologies and reasoners with clouds. Advances in Civil Engineering. Article ID 5478381.

https://doi.org/10.1155/2018/5478381

Koch, C., Vonthron, A., \& Konig, M. (2017). A tunnel information modelling framework to support management, simulations and visualisations in mechanised tunnelling projects. Automation in Construction, 83, 78-90.

https://doi.org/10.1016/j.autcon.2017.07.006

Lai, J. X., Qiu, J. L., Chen, J. X., Wang, Y. Q., \& Fan, H. B. (2014). Application of wireless intelligent control system for HPS lamps and LEDs combined illumination in road tunnel. Computational Intelligence and Neuroscience. Article ID 429657. https://doi.org/10.1155/2014/429657

Lai, J. X., Wang, X. L., Qiu, J. L., Chen, J. X., Hu, Z. N., \& Wang, H. (2018). Extreme deformation characteristics and countermeasures for a tunnel in difficult grounds in southern Shaanxi, China. Environmental Earth Sciences, 77, 706. https://doi.org/10.1007/s12665-018-7888-2

Lee, P. C., Wang, Y. H., Lo, T. P., \& Long, D. B. (2018). An integrated system framework of building information modelling and geographical information system for utility tunnel maintenance management. Tunneling and Underground Space Technology, 79, 263-273.

https://doi.org/10.1016/j.tust.2018.05.010

Lee, S., Tae, S., Roh, S., \& Kim, T. (2015). Green template for life cycle assessment of buildings based on building information modeling: Focus on embodied environmental impact. Sustainability, 7, 16498-16512.

https://doi.org/10.3390/su71215830

Liu, S., Meng, X. H., \& Tam, C. M. (2015). Building information modeling based building design optimization for sustainability. Energy and Buildings, 105, 139-153.

https://doi.org/10.1016/j.enbuild.2015.06.037

Liu, X. R., Han, Y. F., Li, D. L., Tu, Y. L., Deng, Z. Y., Yu, C. T., \& Wu, X. C. (2019). Anti-pull mechanisms and weak interlayer parameter sensitivity analysis of tunnel-type anchorages in soft rock with underlying weak interlayers. Engineering Geology, 253, 123-136. https://doi.org/10.1016/j.enggeo.2019.03.012

Lu, Y. J., Wu, Z. L., Chang, R. D., \& Li, Y. K. (2017). Building Information Modeling (BIM) for green buildings: A critical review and future directions. Automation in Construction, 83, 134-148. https://doi.org/10.1016/j.autcon.2017.08.024

Macher, H., Landes, T., \& Grussenmeyer, P. (2017). From point clouds to building information models: $3 \mathrm{D}$ semi-automatic reconstruction of indoors of existing buildings. Applied Sciences, 7, 1030. https://doi.org/10.3390/app7101030 
Mahdjoubi, L., Moobela, C., \& Laing, R. (2013). Providing realestate services through the integration of 3D laser scanning and building information modelling. Computers in Industry, 64, 1272-1281. https://doi.org/10.1016/j.compind.2013.09.003

Martinez-Aires, M. D., Lopez-Alonso, M., \& Martinez-Rojas, M. (2018). Building information modeling and safety management: A systematic review. Safety Science, 101, 11-18. https://doi.org/10.1016/j.ssci.2017.08.015

Marzouk, M., Abdelaty, A. (2014). Monitoring thermal comfort in subways using building information modeling. Energy and Buildings, 84, 252-257.

https://doi.org/10.1016/j.enbuild.2014.08.006

Marzouk, M., Azab, S., \& Metawie, M. (2016). Framework for sustainable low-income housing projects using building information modeling. Journal of Environmental Informatics, 28, 25-38. https://doi.org/10.1016/j.enbuild.2014.08.006

Peng, C. H., Wu, X. (2015). Case study of carbon emissions from a building's life cycle based on BIM and Ecotect. Advances in Materials Science and Engineering. Article ID 954651. https://doi.org/10.1155/2015/954651

Porwal, A., \& Hewage, K. N. (2012). Building information modeling-based analysis to minimize waste rate of structural reinforcement. Journal of Construction Engineering and Management, 138, 943-954. https://doi.org/10.1061/(ASCE)CO.1943-7862.0000508

Porwal, A., \& Hewage, K. N. (2013). Building Information Modeling (BIM) partnering framework for public construction projects. Automation in Construction, 31, 204-214. https://doi.org/10.1016/j.autcon.2012.12.004

Reychav, I., Leitan, R. M., \& McHaney, R. (2017). Sociocultural sustainability in green building information modeling. Clean Technologies and Environmental Policy, 19, 2245-2254. https://doi.org/10.1007/s10098-017-1409-y

Ryu, D. W., Kim, J. I., Suh, S., and Suh, W. (2015). Evaluating risks using simulated annealing and Building Information Modeling. Applied Mathematical Modelling, 39, 5925-5935. https://doi.org/10.1016/j.apm.2015.04.024

Sanhudo, L., Ramos, N. M. M., Martins, J. P., Almeida, R. M. S. F., Barreira, E., Simoes, M. L., \& Cardoso, V. (2018). Building information modeling for energy retrofitting - A review. Renewable \& Sustainable Energy Reviews, 89, 249-260. https://doi.org/10.1016/j.rser.2018.03.064

Shi, G. L., Song, Z. P., Hu, R. Q., Xiao, K. H., Wang, T., \& Tang, K. Y. (2017). Application and development prospect analysis of BIM in tunnel engineering. In Proceedings of the 2nd National Geotechnical Engineering BIM Technical Seminar (pp. 288-293). Kunming, China.
Song, Z. P., Shi, G. L., Wang, J. B., Zheng, W. J., Wang, J. J., \& Wang, T. (2018). Research on the framework of tunnel cooperative management platform based on BIM technology. Journal of Geotechnical Engineering, 40, 117-121.

Song, Z. P., Yang, T. T., \& Jiang, A. N. (2016). Elastic-plastic numerical analysis of tunnel stability based on the closest point projection method considering the effect of water pressure. Mathematical Problems in Engineering. Article ID 2569345. https://doi.org/10.1155/2016/2569345

Wang, J. B., Li, W. W., \& Song, Z. P. (2019). Development and implementation of new triangular finite element based on MGE theory for bi-material analysis. Results in Physics, 13, 102231. https://doi.org/10.1016/j.rinp.2019.102231

Wang, J. B., Huo, Q, Song, Z. P., \& Zhang, Y. W. (2019). Study on adaptability of primary support arch cover method for largespan embedded tunnels in the upper-soft lower-hard stratum. Advances in Mechanical Engineering, 11(1). https://doi.org/10.1177/1687814018825375

Wong, P. F., Salleh, H., \& Rahim, F. A. M. (2015). A relationship framework for Building Information Modeling (BIM) capability in quantity surveying practice and project performance. Informes de la Construcción, 67. https://doi.org/10.3989/ic.15.007

Xiao, K. H., Song, Z. P., Shi, G. L., Hu, R. Q., Wang, T., \& Tang, K. Y. (2017). BIM technology and its application in tunnel engineering. In Proceedings of the 2nd National Geotechnical Engineering BIM Technical Seminar (pp. 280-287). Kunming, China.

Yalcinkaya, M., \& Singh, V. (2015). Patterns and trends in Building Information Modeling (BIM) research: A latent semantic analysis. Automation in Construction, 59, 68-80. https://doi.org/10.1016/j.autcon.2015.07.012

Zhang, S. J., Teizer, J., Lee, J. K., Eastman, C. M., \& Venugopal, M. (2013). Building Information Modeling (BIM) and safety: Automatic safety checking of construction models and schedules. Automation in Construction, 29, 183-195. https://doi.org/10.1016/j.autcon.2012.05.006

Zhang, S. R., Pan, F., Wang, C., Sun, Y. J., \& Wang, H. X. (2017). BIM-based collaboration platform for the management of EPC projects in hydropower engineering. Journal of Construction Engineering and Management, 143(12), 04017087. https://doi.org/10.1061/(ASCE)CO.1943-7862.0001403

Zhou, W. H., Qin, H. Y., Qiu, J. L., Fan, H. B., Lai, J. X., Wang, K., \& Wang, L. X. (2017). Building information modelling review with potential applications in tunnel engineering of China. Royal Society Open Science, 4, 170174.

https://doi.org/10.1098/rsos.170174 